\title{
Oral Cancer Early Detection - a Pressing Need for Continuing Education in Jordan
}

\author{
Y Hassona ${ }^{1 *}$, F Sawair ${ }^{1}$, Z Baqain ${ }^{1}$, W Maayta ${ }^{1}$, A Shahin ${ }^{1}$, C Scully ${ }^{2}$
}

\begin{abstract}
Aims: To identify deficiencies in the knowledge of general medical (GMPs) and dental practitioners (GDPs) regarding early detection of oral cancer, to compare differences between subgroups, and to identify educational needs for continuing education courses on this subject. Materials and Methods: 165 GDPs and 165 GMPs were directly interviewed to assess their knowledge about oral cancer using a pre-tested survey. Knowledge scores were generated from correct responses of participants to factual questions about oral cancer. Results: Few participants (15.2\% of GDPs and $\mathbf{1 2 . 8 \%}$ of GMPs) reported that they attended any continuing education courses on oral cancer during their professional career. Most participants (81.6\% of GDPs and 86.3\% of GMPs) admitted that their knowledge about oral cancer was not up to date and that they would need to attend continuing education courses on oral cancer if available. A few significant differences were identified between groups regarding knowledge about risk factors, clinical presentations and diagnostic techniques but the overall knowledge scores showed no significant difference between GMPs and GDPs. Conclusions: The present study identified several deficiencies in the knowledge of GMPs and GDPs regarding risk factors, clinical presentations, and diagnostic techniques of oral cancer. Interventions to correct these defects through properly designed continuing education courses are required.
\end{abstract}

Keywords: Oral - cancer - continuing education - early detection - prevention

Asian Pac J Cancer Prev, 16 (17), 7727-7730

\section{Introduction}

Oral cancer is an important health issue with a continuing worldwide increase in incidence; especially in the Indian subcontinent (Krishna et al., 2013). More than 500,000 patients are estimated to have oral cancer globally with approximately 389,000 new cases per annum (Ferlay et al., 2008). Oral cancer is relatively uncommon in the Arab world (Zini et al., 2012, Ismail et al., 2013); the age standardized incidence rates in Jordan are 1.5 in men and 0.8 in women (Ferlay et al., 2013; Ismail et al., 2013). Early diagnosis and treatment minimise morbidity and mortality but most oral cancers even in developed countries are detected at late stages, resulting in a low 5-year survival rate (approximately 50\%) (La Vecchia et al., 2004).

Tobacco and alcohol are the two main identifiable risk factors for oral cancer (Alsanosy, 2014; Quadri et al., 2015). About $30 \%$ of the Jordanian population smoke tobacco in the form of cigarettes or narghile - a tobacco pipe that draws the smoke through water, and around $15 \%$ consume alcohol regularly (Hassona et al., 2015). Early-stage oral cancers are often asymptomatic and present clinically as a white or red patch, ulcer, or lump. Emphasis, therefore, has been placed on opportunistic screening of the oral mucosa by medical and dental practitioners as a proactive preventative measure which may lead to reduced morbidity and mortality from oral cancer (Warnakulasuriya et al., 1991; Hassona et al., 2014; Hassona et al., 2015). In addition, patients with oral lesions are often first seen by general practitioners, both medical and dental. Therefore, general practitioners are in a unique position to detect oral cancer at early stages (Kujan and Sloan, 2013).

Inadequate knowledge about oral cancer has been widely documented among general practitioners from both developed and developing countries (Horowitz et al., 1996; Carter and Ogden, 2007; Jaber et al., 2011; Alaizari and Al-Maweri; Saleh et al., 2014), and is one factor that contributes to delays in diagnosis, referral, and treatment leading to sub-optimal management (Saleh et al., 2014). Continuing education courses can have a positive influence on practitioners ability to detect oral cancer at early stages through improving their knowledge and early detection practices (Silverman et al., 2010). Indeed, continuing education is mandatory in some countries, such as UK and USA.

We have recently demonstrated an inadequate level of knowledge, attitudes, and practices of primary health care professionals from Jordan with regard to oral cancer diagnosis (Hassona et al., 2015). In the present report, we performed further analyses to assess the educational needs of general medical practitioners (GMPs) and general dental practitioners (GDPs) from Jordan with 


\section{Y Hassona et al}

regard to oral cancer, and identify any deficiencies in their knowledge in order to help develop an oral cancer continuing education module tailored to the specific educational needs of Jordanian general medical and dental practitioners.

\section{Materials and Methods}

We randomly selected a sample of 190 licensed
GDPs and 190 licensed GMPs from the registries of the Jordanian Dental and Medical Associations to participate in the study. After eliminating all ineligible practitioners (not interested, moved out of country, or busy at the survey time), a final sample of 330 general practitioners (165 medical and 165 dental practitioners) was obtained. Sample size was calculated using nQuery Advisor Software with oversampling to account for possible withdrawals. The characteristics of those who

Table 1. A comparison between the knowledge of GMPs and GDPs about oral cancer

\begin{tabular}{|c|c|c|c|c|c|}
\hline \multirow{2}{*}{ Variable } & \multicolumn{2}{|c|}{ Correct responses of GDPs } & \multicolumn{2}{|c|}{ Correct responses of GMPs } & \multirow{2}{*}{$\mathrm{P}$ value* } \\
\hline & No. & $\%$ & No. & $\%$ & \\
\hline \multicolumn{6}{|l|}{ Knowledge about risk factors } \\
\hline Tobacco & 161 & 97.6 & 158 & 95.8 & 0.36 \\
\hline Alcohol & 106 & 64.2 & 83 & 50.3 & 0.01 \\
\hline HPV & 60 & 36.4 & 83 & 50.3 & 0.01 \\
\hline Sun exposure & 50 & 30.3 & 30 & 18.2 & 0.01 \\
\hline Poor diet & 36 & 21.8 & 52 & 31.5 & 0.046 \\
\hline Advanced age & 44 & 26.7 & 79 & 47.9 & $<0.001$ \\
\hline Immunosuppression & 71 & 43 & 95 & 57.6 & 0.008 \\
\hline Presence of OPMDs & 100 & 60.6 & 114 & 69.1 & 0.12 \\
\hline Chewing habits & 89 & 53.9 & 89 & 53.9 & 1 \\
\hline Previous history of oral cancer & 124 & 75.2 & 119 & 72.1 & 0.53 \\
\hline \multicolumn{6}{|l|}{ Knowledge about OPMDs } \\
\hline Leukoplakia & 118 & 71.5 & 101 & 61.2 & 0.048 \\
\hline Erythroplakia & 88 & 53.3 & 58 & 35.2 & 0.001 \\
\hline Lichenoid lesions & 47 & 28.5 & 46 & 27.9 & 0.9 \\
\hline Chronic hyperplastic candidiasis & 70 & 42.4 & 61 & 37 & 0.31 \\
\hline Actinic cheilitis & 36 & 21.8 & 23 & 13.9 & 0.062 \\
\hline Oral sub-mucous fibrosis & 55 & 33.3 & 62 & 37.6 & 0.42 \\
\hline \multicolumn{6}{|l|}{ Knowledge about clinical presentations } \\
\hline Persistent ulcer & 113 & 68.5 & 110 & 66.7 & 0.72 \\
\hline Red lesion & 98 & 59.4 & 73 & 44.2 & 0.006 \\
\hline White lesion & 103 & 62.4 & 81 & 49.1 & 0.015 \\
\hline Lump & 97 & 58.8 & 112 & 67.9 & 0.087 \\
\hline Non healing socket & 63 & 38.2 & 79 & 47.9 & 0.075 \\
\hline Enlarged lymph nodes & 118 & 71.5 & 119 & 72.1 & 0.9 \\
\hline Dysphagia \& limited tongue mobility & 100 & 60.6 & 122 & 73.9 & 0.01 \\
\hline \multicolumn{6}{|l|}{ Knowledge about epidemiology } \\
\hline Males & 74 & 44.8 & 108 & 65.5 & $<0.001$ \\
\hline Older age groups ( $>40$ years) & 135 & 81.8 & 130 & 78.8 & 0.68 \\
\hline \multicolumn{6}{|l|}{ Knowledge about diagnostic techniques } \\
\hline Scalpel biopsy & 140 & 84.8 & 121 & 73.3 & 0.01 \\
\hline Toluidine blue & 40 & 24.2 & 29 & 17.6 & 0.14 \\
\hline Brush biopsy & 47 & 28.5 & 71 & 43 & 0.006 \\
\hline Fluorescent imaging & 113 & 68.5 & 128 & 77.6 & 0.063 \\
\hline Exfoliative cytology & 77 & 46.7 & 93 & 56.4 & 0.078 \\
\hline
\end{tabular}

Overall knowledge score $($ mean $\pm \mathrm{SD}) * *$

*P value of Chi Square test. **Knowledge score ranges from 0-31. ***P value of Student's t-test. SD: standard deviation. OPMDs: oral potentially malignant disorders 
were selected were comparable to that of the national health workforce profiles (Department of Statistics, 2015).

After explaining the purpose of the study, and reassurance of participants that no personal identifying information would be disclosed, two trained coauthors (AS and WM) conducted a pretested and validated face to face interviews of the recruited practitioners to assess knowledge related to early diagnosis of oral cancer, confidence with oral cancer diagnosis, and the need for continuing professional development courses on oral cancer. There were 31 items on the survey to assess knowledge about risk factors and epidemiology, oral lesions/conditions that might transform to cancer, clinical signs and symptoms of oral cancer, and techniques that might help in early detection of oral cancer. This study was reviewed and approved by The University of Jordan, Faculty of Dentistry Research and Ethics Committee.

The responses were coded and analyzed using SPSS for Windows release 16.0 (SPSS Inc., Chicago, IL, USA) by calculating frequencies for categorical data and means for continuous data. Each correct response to knowledge questions was given a score of 1 point and "knowledge score" was calculated for each respondent by summating all the scores. Score 31 indicated that the participant answered all the factual questions about oral cancer correctly, and score zero indicated that the participant failed to answer any question correctly or answered "I do not know" for all questions. Chi Square test and Student's $\mathrm{t}$-test were used to examine differences between groups. A $\mathrm{P}$-value of $<0.05$ was considered statistically significant.

\section{Results}

The respondents consisted of 233 males $(70.6 \%)$ and 97 females $(29.4 \%)$ with a mean age of 37.6 years \pm 11.8 and a mean years of clinical practice of 12.6 years \pm 11.2 . Few participants (15.2\% of GDPs and $12.8 \%$ of GMPs) reported that they attended a continuing education course on oral cancer during their professional career. Most participants (81.6 of GDPs and 86.3\% OF GMPs) admitted that their knowledge about oral cancer was not up to date and that they need to attend continuing education courses on oral cancer if available.

The majority knew that squamous cell carcinoma is the most common type of oral cancer with $93.2 \%$ of GDPs and $90.1 \%$ of GMPs answering correctly. Tobacco smoking was known as a main risk factor for oral cancer by most respondents from both groups (97.6\% of GDPs and $95.8 \%$ of GMPs). On the contrary, alcohol use was less likely to be identified as a risk factor for oral cancer by both groups, but GDPs were more likely to respond correctly $(\mathrm{p}<0.05)$. Significantly more GMPs knew about human papilloma virus (HPV), prolonged sun exposure, prolonged immunosuppression, poor diet and advanced age as risk factors of oral cancer $(\mathrm{p}<0.05)$. Presence of oral potentially malignant disorders (OPMDs) was correctly identified as a risk factor for oral cancer by $60.6 \%$ of GDPs and $69.1 \%$ of GMPs, with no significance between groups. Almost equal percentages of GMPs and GDPs correctly identified chewing habits and a history of cancer in the aerodigestive tract as risk factors of oral cancer (Table 1).
Significantly more GMPs knew that oral cancer is more common in males $(\mathrm{p}<0.05)$. Most participants from both groups knew that oral cancer is more likely to affect older patients, with no significance between GMPs and GDPs.

Leukoplakia followed by erythroplakia were the best known OPMDs in both groups, but GDPs were more likely to respond correctly $(\mathrm{p}<0.05)$ than GMPs. Other OPMDs, including lichenoid lesions, chronic hyperplastic candidiosis, actinic chelitis, oral submucous fibrosis, were much less recognised for their risk of malignant transformation by GMPs and GDPs, with no significance between groups (Table 1).

Enlargement of cervical lymph nodes was the best known clinical presentation of oral cancer by GMPs and GDPs, with no significant between groups. GDPs were more aware of the fact that oral cancer might present clinically as a red or white mucosal patch $(p<0.05)$, while GMPs were more aware about dysphagia and limited tongue mobility as possible signs of oral cancer $(\mathrm{p}<0.05)$. Other possible presentations of oral cancer including, non-healing extraction socket, persistent ulcer, and lump, were known by almost equal percentages of GMPs and GDPs (Table 1).

When asked about diagnostic techniques that might help in early detection of oral cancer: most GDPs reported scalpel biopsy and most GMPs reported fluorescent imaging. Other diagnostic techniques including toluidine blue staining of oral lesions, brush biopsy, and exfoliative cytology were less known to both GMPs and GDPs, with no significance between groups (Table 1).

When overall knowledge scores about oral cancer were calculated, no significant differences were found between GMPs and GDPs (Table 1).

\section{Discussion}

Attendance at continuing education courses has a positive influence on practitioners ability to detect oral cancer at early stages through improving their knowledge and early detection practices (Silverman et al., 2010). The majority of GMPs and GDPs in the present study admitted that their knowledge about oral cancer was not up to date and that there is a need for them to attend continuing education courses. Nevertheless, only few participants reported their attendance at continuing education courses dedicated to oral cancer during their professional career. This could be explained by the paucity of such courses in the Middle East in general, and in Jordan in particular. In a previous study, when asked to specify which courses they need to have continuing education, Jordanian GDPs requested courses in dental implantology, orthodontics and endodontics, but few requested courses to improve knowledge in oral cancer (Sawair and Baqain, 2004).

The findings of the present studies identified several deficiencies in the knowledge of Jordanian GMPs and GDPs with regard to oral cancer. Similar to other studies we found that the association between alcohol use and oral cancer is less known to general practitioners (Horwitz et al., 1996; LeHew et al., 2010; Jaber et al., 2011). Continuing education courses should therefore focus on the adverse effects of alcohol on oral tissues including 


\section{$Y$ Hassona et al}

the increased risk of oral cancer development. This is particularly important because alcohol use is becoming more popular in conservative communities including those of the Middle East. GDPs and GMPs in the present study appeared not much aware about the association between oral cancer and HPV, sun exposure, immunosuppression, chewing habits, and advanced age. This could limit their ability to identify at risk patients and take appropriate actions.

Some oral cancers are preceded by noticeable mucosal changes known as OPMDs (Hassona et al., 2014; Scully, 2014). Leukoplakia and erythroplakia are the best known OPMDs, although other diseases such as oral lichenoid lesions, chronic hyperplastic candidiosis, and submucous fibrosis might also transform to cancer. GDPs in the present study were significantly more aware about the significance of erythroplakia and leukoplakia as OPMDs compared to GMPs. Efforts therefore should be placed to educate GMPs about such lesions. Both GDPs and GMPs in the present study were less aware about early clinical presentations of oral cancer such as white or red mucosal changes. Most participants, however, were able to identify latter signs of oral cancer such as enlarged cervical lymph nodes, dysphagia, and limited tongue mobility, indicating a defect in their knowledge regarding early manifestations of cancer in the oral cavity. In fact, we have suggested a "RULE" for cancer diagnosis - an acronym based on Red or white lesion, Ulcer, Lump, Exceeding 3 weeks duration to help practitioners to identify suspicious mucosal changes that require urgent referral (Scully, 2014).

Several diagnostic techniques have been proposed to help in screening and early detection of oral cancer (Scully et al., 2008; Masthan et al., 2012). Scalpel biopsy, however, is considered the gold standard in oral cancer diagnosis. Most GDPs and GMPs were aware of this fact, but fewer practitioners knew about other less invasive and potentially useful techniques such as toluidine blue staining, fluorescent imaging, exfoliative cytology, and brush biopsy. These techniques however are not universally available, have variable diagnostic yields, and their use by general practitioners requires further training and experience.

The present study identified several deficiencies in the knowledge of Jordanian GMPs and GDPs regarding risk factors, clinical presentations, and diagnostic techniques of oral cancer. Interventions to correct these defects through properly designed continuing education courses are required.

\section{References}

Alaizari NA, Al-Maweri SA (2014). Oral cancer: knowledge, practices and opinions of dentists in yemen. Asian Pac J Cancer Prev, 15, 5627-31.

Alsanosy RM (2014). Smokeless tobacco (shammah) in saudi arabia: a review of its pattern of use, prevalence, and potential role in oral cancer. Asian Pac J Cancer Prev. 15, 6477-83.

Carter LM, Ogden GR (2007). Oral cancer awareness of general medical and general dental practitioners. Br Dent J.203, 10 . Department of Statistics. Hashemite Kingdome J.

Ferlay J, Shin HR, Bray F, et al (2010). Estimates of worldwide burden of cancer in 2008: GLOBOCAN 2008. Int J Cancer. 127, 2893-917.

Ferlay J, Soerjomataram I, Ervik M, et al (2013). GLOBOCAN 2012 v1.0, Cancer incidence and mortality worldwide: IARC Cancer Base No. 11. Lyon, France: International Agency for Research on Cancer.

Hassona Y, Scully C, Abu Ghosh M, et al (2015). Mouth cancer awareness and beliefs among dental patients. Int Dent $J$. 65, 15-21.

Hassona Y, Scully C, Almangush A, et al (2014). Oral potentially malignant disorders among dental patients: a pilot study in Jordan. Asian Pac J Cancer Prev, 15, 10427-31.

Hassona Y, Scully C, Shahin A, et al (2015). Factors influencing early detection of oral cancer by primary health-care professionals. J Cancer Educ.

Horowitz AM, Goodman HS, Yellowitz JA, et al (1996). The need for health promotion in oral cancer prevention and early detection. J Public Health Dent, 56, 319-330.

Ismail SI, Soubani M, Nimri JM, et al (2013). Cancer incidence in Jordan from 1996 to 2009--a comprehensive study. Asian Pac J Cancer Prev, 14, 3527-34

Jaber L, Shaban S, Hariri D, et al (2011). Perceptions of health care practitioners in Saudi Arabia regarding their training in oral cancer prevention and early detection. Int $J$ Health Care Qual Assur, 24, 8-18.

Krishna Rao SV, Mejia G, Roberts-Thomson K, et al (2013). Epidemiology of oral cancer in Asia in the past decade--an update (2000-2012). Asian Pac J Cancer Prev, 14, 5567-77.

Kujan O, Sloan P (2013). Dilemmas of oral cancer screening: an update. Asian Pac J Cancer Prev, 14, 3369-73

La Vecchia C, Lucchini F, Negri E, et al (2004). Trends in oral cancer mortality in Europe. Oral Oncol, 40, 433-9

LeHew CW, Epstein JB, Kaste LM, et al (2010). Assessing oral cancer early detection: clarifying dentists' practices.J Public Health Dent, 70, 93-100.

Masthan KM, Babu NA, Dash KC, et al (2012). Advanced diagnostic aids in oral cancer. Asian Pac J Cancer Prev, 13, 3573-6.

Quadri MF, Alharbi F, Bajonaid AM, et al (2015). Oral squamous cell carcinoma and associated risk factors in jazan, saudi arabia: a hospital based case control study. Asian Pac J Cancer Prev, 16, 4335-8.

Saleh A, Kong YH, Vengu N, et al (2014). Dentists' perception of the role they play in early detection of oral cancer. Asian Pac J Cancer Prev, 15, 229-37.

Sawair FA, Baqain ZH (2004). Continuing education: views of Jordanian general dental practitioners. Cairo Dental J, 20, 399-402.

Scully C (2014). Challenges in predicting which oral potentially malignant diseases will progress to neoplasia. Oral Dis. 20, $1-5$.

Scully C, Bagan JV, Hooper C, et al (2008). Oral cancer: current and future diagnostic techniques. Am J Dent, 21, 199-209.

Silverman S Jr, Kerr AR, Epstein JB (2010). Oral and pharyngeal cancer control and early detection. J Cancer Educ, 25, 279-81.

Warnakulasuriya KA, Johnson NW (1999). Dentists and oral cancer prevention in the UK: opinions, attitudes and practices to screening for mucosal lesions and to counselling patients on tobacco and alcohol use: baseline data from 1991. Oral Dis, 5, 10-4.

Zini A, Nasser N, Vered Y (2012). Oral and pharyngeal cancer among the Arab population in Israel from 1970 to 2006. Asian Pac J Cancer Prev, 13, 585-9. 